\title{
LE DROIT À LA SANTÉ: UN DROIT FONDAMENTAL AUSSI AU TRAVAIL?
}

\author{
EL DERECHO A LA SALUD: ¿UN DERECHO \\ FUNDAMENTAL TAMBIÉN EN EL LUGAR DE \\ TRABAJO?
}

\begin{abstract}
Loïc Lerouge ${ }^{1}$
Recibido: 28/12/2019

Aceptado: 20/01/2020

SUMARIO: I. El derecho a la salud en el trabajo: reconocimiento y desafíos. 1. Los vínculos entre el derecho a la salud en el trabajo y el derecho de la salud en el trabajo. 2. El derecho a la salud en el trabajo: ¿un derecho fundamental? II. El derecho a la protección de la salud en el trabajo. 1. Introducir la prevención en el derecho a la salud en el trabajo. 2. Derecho a la salud en el trabajo y enfoque ético.
\end{abstract}

SOMMAIRE: I. Le droit à la santé au travail: reconnaissance et enjeux. 1. Les liens entre droit à la santé au travail et droit de la santé au travail. 2. Le droit à la santé au travail : un droit fondamental ? II. Le droit à la protection de la santé au travail. 1. Introduire de la prévention dans le droit à la santé au travail. 2. Droit à la santé au travail et approche éthique.

Resumen: El derecho a la salud en el trabajo, ¿debe ser considerado como un derecho fundamental o bien el derecho fundamental a la salud se aplica directamente al trabajo? El enfoque de la salud en el trabajo bajo el prisma de los textos internacionales relativos a los derechos fundamentales y a los derechos del hombre, así como la evolución bajo la mirada del reconocimiento de la salud mental en el trabajo, junto a la salud física en relación particular al desarrollo de los riegos organizacionales, permiten interrogarnos sobre el alcance del derecho fundamental a la salud en el ámbito del trabajo. Se trata de una cuestión de gran importancia hoy en día, en particular bajo el prisma del reconocimiento de los riesgos psicosociales en el trabajo, pero igualmente los que afectan a la salud mental en el trabajo, especialmente en razón de las organizaciones del trabajo que hoy impactan, cada vez más, en la salud mental de los trabajadores. Para responder a estos interrogantes, el reconocimiento y las cuestiones relacionadas al derecho a la salud en el trabajo deben ser explicitadas y precisadas. Estos desafíos se proyectan en el objetivo de garantizar una mejor protección de la salud física y mental en el trabajo y de sentar un enfoque preventivo que permita integrar, de hoy en adelante, cuestiones de orden ético que deben considerarse necesarias. Las páginas que siguen están dedicadas al reconocimiento y al rol que desempeña el derecho a la salud en el trabajo y a su cualificación o no como derecho fundamental.

Palabras clave: Derecho fundamental, salud, trabajo, riesgos psicosociales, prevención de riesgos profesionales.

Résumé: Le droit à la santé au travail doit-il être considéré comme un droit fondamental ou bien le droit fondamental à la santé s'applique-t-il directement au travail ? L'approche de la santé au travail au prisme des textes internationaux relatifs aux droits fondamentaux

${ }^{1}$ Chargé de recherche CNRS. COMPTRASEC UMR 5114. CNRS - Université de Bordeaux. 
et aux droits de l'Homme, aussi l'évolution au regard de la reconnaissance de la santé mentale au travail au côté de la santé physique au regard notamment de l'essor des risques organisationnels, amènent à nous interroger sur la portée du droit fondamental à la santé à l'aune du travail. C'est une question de grande importance aujourd'hui, notamment au regard de la reconnaissance des risques psychosociaux au travail, mais également des atteintes à la santé mentale au travail notamment en raison d'organisations du travail qui aujourd'hui impactent de plus en plus la santé mentale des travailleurs. Pour répondre à ces interrogations, la reconnaissance et les enjeux liés au droit à la santé au travail doivent être explicités et précisés. Ces enjeux se prolongent dans l'objectif de garantir une meilleure protection de la santé physique et mentale au travail et d'asseoir une approche préventive tout en intégrant dorénavant de nécessaires questionnements de l'ordre de l'éthique. Les pages suivantes sont dédiées à la reconnaissance et aux enjeux du droit à la santé au travail et à sa qualification ou non comme droit fondamental.

Mots clé: Droit fondamental, santé, travail, risques psychosociaux, prévention des risques professionnels.

Le point de départ de la réflexion est parti de l'adoption par l'ONU le 23 septembre 2019 de la résolution relative à la «Protection des droits de travailleurs exposés à des substances et déchets dangereux» ${ }^{2}$. Cette résolution et sa conclusion étaient inscrites dans le point 3 de l'ordre du jour de la 42 session du Conseil des Droits de l'Homme relatif à la «Promotion et protection de tous les droits de l'homme, civils, politiques, économiques, sociaux et culturels, y compris le droit au développement». Or, même si le texte porte sur la protection contre les produits chimiques au travail, l'Assemblée Générale de l'ONU rappelle avec force et instamment dans la conclusion de sa résolution la nécessité de renforcer «le cadre mondial de gestion des produits chimiques afin de prévenir et réduire au minimum l'exposition risquée aux substances dangereuses, de promouvoir le droit de chacun, y compris des travailleurs, de jouir du meilleur état de santé physique et mentale possible, et des conditions de travail justes et favorables».

La lecture de la résolution permet de prendre conscience combien le droit international et les droits fondamentaux offrent un cadre juridique garant de la protection de la santé physique et mentale au travail. Ainsi, en matière de santé mentale au travail, L’Organisation Mondiale de la Santé (OMS) invite à «combattre les problèmes de santé mentale, quelle qu'en soit la cause» ${ }^{3}$, la santé mentale étant une composante essentielle de la santé. L'objectif est de crear un entorno saludable de trabajo qui permet d'atteindre ceux de la définition de la santé mentale de l'OMS selon laquelle «La santé mentale est un état de bien-être dans lequel une personne peut se réaliser, surmonter les tensions normales de la vie, accomplir un travail productif et contribuer à la vie de sa communauté. Dans ce sens positif, la santé mentale est le fondement du bien-être d'un individu et du bon fonctionnement d'une communauté» ${ }^{4}$. Les textes internationaux qui fondent le

\footnotetext{
${ }^{2}$ Naciones Unidas, Consejo de Derechos Humanos, resolución sobre la Protección de los derechos de los trabajadores expuestos a sustancias y desechos peligrosos, 23 de septiembre 2019, HRC/42/L.27 https://undocs.org/es/A/HRC/42/L.27.

3 Hoja informativa sobre la "Salud mental en el lugar de trabajo", Mayo de 2019, https://www.who.int/mental health/in the_workplace/es/.

${ }^{4}$ OMS, Salud mental: fortalecer nuestra respuesta, 30 de marzo de 2018, https://www.who.int/es/newsroom/fact-sheets/detail/mental-health-strengthening-our-response.
} 
caractère fondamental de la protection de la santé physique et mentale au travail sont nombreux, mais finalement peu évoqués. La résolution de l’ONU du 23 septembre 2019 permet ainsi de rappeler que pour que soient respectés des textes fondamentaux tels que notamment la Charte des Nations Unies, la Déclaration universelle des droits de l'homme, le Pacte international relatif aux droits économiques, sociaux et culturels, le Pacte international relatif aux droits civils et politiques, il est nécessaire de réduire l'effet néfaste sur la santé physique et mentale l'exposition aux substances dangereuses sur le lieu du travail et de mettre l'accent sur les stratégies préventives primaires.

$\mathrm{Au}$ regard du Pacte international relatif aux droits économiques, sociaux et culturels, de la Constitution de l'Organisation internationale du Travail et de la Déclaration de Philadelphie concernant les buts et objectifs de l'Organisation internationale du Travail (OIT), l'exercice du droit de chaque travailleur de jouir du meilleur état de santé physique et mentale doit être garanti.

L'approche de la santé au travail au prisme des textes internationaux relatifs aux droits fondamentaux et aux droits de l'Homme, mais aussi l'évolution au regard de la reconnaissance de la santé mentale au travail au côté de la santé physique au regard notamment de l'essor des risques organisationnels, amènent à nous interroger sur la portée du droit fondamental à la santé à l'aune du travail. En d'autres termes, le droit à la santé au travail doit-il être considéré comme un droit fondamental ou bien le droit fondamental à la santé s’applique-t-il directement au travail?

La question est d'importance aujourd'hui, notamment au regard de la reconnaissance des risques psychosociaux au travail, mais également des atteintes à la santé mentale au travail notamment en raison d'organisations du travail qui aujourd'hui impactent de plus en plus la santé mentale des travailleurs. Pour répondre à ces interrogations, la reconnaissance et les enjeux liés à au droit à la santé au travail doivent être explicités et précisés (I). Ces enjeux se prolongent dans l’objectif de garantir une meilleure protection de la santé physique et mentale au travail et d'asseoir une approche préventive tout en intégrant dorénavant de nécessaires questionnements de l'ordre de l'éthique (II).

\section{LE DROIT A LA SANTE AU TRAVAIL: RECONNAISSANCE ET ENJEUX}

Traditionnellement se confrontent le droit à la santé et le droit de la santé stricto sensu. Ces deux approches juridiques de la santé n'opèrent pas de distinction entre santé physique et santé mentale, le terme "santé » étant suffisant en lui-même pour englober ces deux volets. Dans le champ du travail, le droit de la santé au travail est tout de suite mis en avant, mais il est fondamentalement lié au droit à la santé appliqué au travail ${ }^{5}$. Pour le comprendre, le droit de la santé et le droit de la santé au travail doivent être explicités (A) tout en questionnant la nécessité ou non de reconnaître un droit fondamental à la santé spécifique au travail (B).

\footnotetext{
${ }^{5}$ Voir Lerouge L., «Well-being at Work: a Few Considerations Under a Legal Perspective» in Addabbo T., Ales E., Curzi Y., Senatori I. (Ed.), Well-being at and through Work, Marco Biagi Fundation, 2017, p. 1532.
} 


\section{Les liens entre droit à la santé au travail et droit de la santé au travail}

Le «droit de la santé» est un ensemble de règles poursuivant un même objectif ${ }^{6}$ et applicable aux «activités dont l'objet de restaurer la santé humaine, de la protéger et d'en prévenir les dégradations» ${ }^{7}$. Dit d'une autre manière, le droit de la santé est «l'ensemble des règles juridiques applicables aux actions de santé» ${ }^{8}$. Il est né de «l'aspiration à un droit à la santé» ${ }^{9}$ permettant ainsi de relier entre elles ces deux conceptions juridiques de la santé au travail.

Le droit de la santé a des origines extrêmement anciennes datant des législations antiques concernant la responsabilité des personnes tenant lieu de médecins ${ }^{10}$ ou de guérisseurs ${ }^{11}$. Le droit de la santé est d'abord né de la nécessité de protéger la société contre les risques d'ordre sanitaire, puis de droits individuels issus du principe fondamental de respect de l'intégrité du corps humain tels que le droit de consentir à l'atteinte physique pour des soins thérapeutiques ou d'obtenir réparation d'atteintes préjudiciables $^{12}$.

Le droit de la santé comporte trois catégories de règles. La première comporte des règles de police car il incite, limite et contrôle les actions intervenant en matière de santé. La deuxième catégorie dispose de règles régissant les services publics de manière à fournir des prestations à caractère sanitaire. Enfin, dans une troisième catégorie, le droit de la santé réglemente les relations entre particuliers, notamment entre les malades et les professionnels de santé ${ }^{13}$.

Le droit de la santé présente la particularité d’être profondément marqué par les droits de l'homme. En effet, il est imprégné du droit à la vie, du droit à la santé complété par le droit à la sécurité sociale, du droit au respect de l'intégrité physique, du droit au respect de l'intimité personnelle, du droit de ne pas souffrir, ... Il comporte un caractère d'ordre public en ce qu'il limite les actions humaines qui peuvent mettre en péril l'état sanitaire de la société et qu'il intervient de façon à permettre un développement du potentiel sanitaire «considéré comme une valeur sociale» ${ }^{14}$. Cette caractéristique d’ordre public et interventionniste du droit de la santé implique donc sa présence dans les relations de travail.

Le droit de la santé au travail s'affirme au travers du droit de la prévention ${ }^{15}$ et s'exprime notamment dans l'élaboration de normes techniques et d'un règlement intérieur. Ces caractéristiques mettent en lumière au sein du droit de la santé au travail

\footnotetext{
${ }^{6}$ Casaux-Labrunée L., «Le "droit à la santé"» in Libertés et droits fondamentaux, Dalloz, 6 ème édition, 2000, p. 609-649.

${ }^{7}$ Forges (de) J.-M., Le droit de la santé, PUF, Coll. Que Sais-je?, 2000, p. 7.

${ }^{8}$ Auby J.-M., Le droit de la santé, PUF, Coll. Thémis, 1981, p. 13.

${ }^{9}$ Forges (de) J.-M., op. cit., p. 6.

${ }^{10}$ Auby J.-M., «Droits de l’homme et droit de la santé (en particulier dans le régime juridique des services publics sanitaires)» in Mélanges offerts à Robert-Edouard Charlier, Service public et libertés, Editions de l’Université et de l’Enseignement Moderne, 1981, pp. 673 à 685.

${ }^{11}$ Auby J.-M., Le droit de la santé, PUF, Coll. Thémis, 1981, p. 13.

12 Auby J.-M., «Droits de l’homme et droit de la santé (en particulier dans le régime juridique des services publics sanitaires)» op. cit.

${ }^{13}$ Auby J.-M., Le droit de la santé, PUF, Coll. Thémis, 1981, p. 15-16.

${ }^{14}$ Auby J.-M., «Droits de l’homme et droit de la santé (en particulier dans le régime juridique des services publics sanitaires)» op. cit.

15 Salmon B., «Le droit de la santé et de la sécurité du salarié, vers un droit au travail sûr», thèse droit, Nantes, 2000, p. 216.
} 
deux ordres de dispositions dont l'objet est différent ${ }^{16}$. Le premier ordre est un ensemble de prescriptions qui imposent des mesures très techniques en matière d'hygiène et de sécurité. Le second ordre concerne les organismes créés dans le cadre du travail dont l'action est de veiller et d'assurer l'application des politiques de prévention des atteintes à la santé au travail.

L'exécution du contrat de travail s'accompagne de risques professionnels qui peuvent compromettre la santé des travailleurs qui rend à ce titre l'employeur débiteur de leur santé. Le droit de la santé se transpose ainsi au travail sous la forme d'un «droit de la santé au travail» consistant à réglementer les moyens d'empêcher la survenance d'atteintes à l'intégrité de la personne du travailleur au temps et au lieu de travail et sous la subordination de l'employeur. Ces règles sont applicables aux actions d'hygiène et de sécurité, le but étant d'éviter les accidents et maladies professionnelles.

Les sources juridiques du droit de la santé au travail sont éclatées entre le droit international, le droit communautaire, le droit national et le droit conventionnel. De nos jours il est aussi d'usage de renvoyer de plus en plus fréquemment vers des normes techniques de type ISO $^{17}$ qui créent une norme extra-juridique, c'est-à-dire une «norme préventive en-dehors du processus classique de création des normes juridiques» ${ }^{18}$. L'employeur doit aussi édicter dans l'entreprise des normes de prévention via par exemple le règlement intérieur en droit français qui engage sa responsabilité et s'impose à l'ensemble de la collectivité de travail. Le pouvoir de l'employeur devient réglementaire en édictant des règles qui s'appliqueront dans le domaine de la protection de la santé des travailleurs et qui s'intégreront dans l'ordre juridique étatique. L'effet impératif de cette réglementation fait que l'employeur se trouve lié par des règles qu'il a édictées lui-même et que le juge pourra interpréter comme une source de droit applicable.

Toutefois, la conjugaison du fait que de plus en plus de systèmes juridiques reconnaissent la «santé physique et mentale» au travail dans leurs lois, réglementations ou jurisprudence avec la portée désormais générale de l'obligation de sécurité et de prévention à la charge de l'employeur depuis la transposition dans l'Union Européenne de la Directive-cadre du 12 juin $1989^{19}$ laisse à penser que la protection de la santé au travail acquiert de nos jours une portée plus fondamentale. Le droit de la santé au travail s'articule désormais avec un « droit à la santé au travail » renvoyant aussi plus largement aux conditions de travail.

Le droit à la santé est un droit fondamental reconnu est par le droit international. La Constitution de l'OMS ${ }^{20}$ du 27 octobre 1946 proclame que «Bénéficier du plus haut standard possible de santé constitue un des droits fondamentaux de tout être humain». Le droit à la santé est aussi reconnu comme fondamental par la Déclaration universelle des droits de l'homme et en Europe par la Charte européenne des droits économiques et sociaux. Le droit à la santé est lié à «des droits et des principes consubstantiels à

\footnotetext{
${ }^{16}$ Durand P., Traité de droit du travail, Tome II, Dalloz, 1950, § 293.

17 Voir la norme ISO 45001 santé et sécurité au travail et le projet de norme ISO45003 sur les risques psychosociaux au travail.

18 Salmon B., Le droit de la santé et de la sécurité du salarié, vers un droit au travail sûr, thèse droit, Nantes, 2000, p. 249.

19 Directive-cadre 89/391/CEE du 12 juin 1989 concernant la mise en œuvre de mesures visant à promouvoir l'amélioration de la sécurité et de la santé des travailleurs au travail, JOCE $n^{\circ} L 183$ du 29 juin 1989 p. 1

${ }^{20}$ http://www.who.int/governance/eb/who_constitution_en.pdf
} 
l'Homme» ${ }^{21}$ au rang desquels la dignité tient une place particulière. Les liens entre le droit à la santé et la protection de la dignité de la personne humaine s’établissent au sein de la protection de l'Homme - entendu comme la «personne humaine» dotée d'un corps et d'un esprit - dans son essence ou dans son existence. Il s'agit de ne pas laisser une personne en détresse, en l'occurrence en raison de son état de santé, sous peine de méconnaître son appartenance à l’humanité22. La santé serait une des conditions, sinon la condition, «sans laquelle l'homme ne peut pas donner le meilleur de lui-même, et sans laquelle il est privé des moyens de se réaliser en tant qu'être humain» ${ }^{23}$.

Le droit à la santé transcende ainsi toutes les frontières comme on l'entend aussi concernant le droit inaliénable à la dignité. De ce fait, le droit à la santé peut être considéré comme indissociable du travail. Toutefois, compte tenu des situations de souffrance qui se développent au travail, individuellement et collectivement parfois ${ }^{24}$, la question de la nécessité ou non de reconnaître formellement un droit fondamental à la santé spécifique au travail peut se poser.

\section{Le droit à la santé au travail: un droit fondamental?}

L’un des enjeux relevant de la reconnaissance par le droit de la santé mentale en droit du travail est de reconnaître le travailleur comme une personne pleine et entière, en d'autres termes que son corps et son esprit sont indissociables. La personne entrant dans une relation contractuelle de travail ne dissocie en effet pas l'aspect mental de sa santé de sa santé physique. La santé fait partie intégrante de la personne humaine qui se réalise aussi au travail et par le travail. Les perspectives ouvertes par cette évolution font progresser le droit de la prévention au travail vers une «nouvelle prévention» plus complète envers la personne du travailleur, mais aussi plus complexe. Le droit à la santé justifie également la création sur les lieux d'exécution du travail de services chargés de prévenir toute altération à la santé des travailleurs. Autrement dit, le but est de protéger la santé de la personne placée dans un contexte particulier - c'est-à-dire dans une situation de subordination et dans l'engrenage de la mise en œuvre d'une organisation - et d'éviter les abus ou les risques qui peuvent en résulter.

L'enjeu est aussi de changer de paradigme en mettant l'accent sur la prévention primaire plutôt que de rester concentré sur la réparation. Les principes de l' «adaptation du travail à l'homme», de "combattre les risques à la source», pourtant reconnus depuis la transposition de la directive-cadre du 12 juin 1989 comme principes généraux de prévention essentiels, doivent marquer de leur empreinte l'approche des conditions de travail, l'élaboration et la mise en œuvre des organisations du travail, la recherche de solutions dans l'innovation. Il en résulte que tous les acteurs du travail sont concernés et doivent participer à la prévention des risques liés à la santé physique et mentale.

\footnotetext{
${ }^{21}$ Mathieu B., «La protection du droit à la santé par le juge constitutionnel. À propos et à partir de la décision de la Cour constitutionnelle italienne $n^{\circ} 185$ du 20 mai 1998», Les Cahiers du Conseil Constitutionnel, $\mathrm{n}^{\circ}$ 6, 1999, p. 59-67.

22 Ibid.

${ }^{23}$ Bedjaoui M., «Le droit à la santé, espoirs, réalités, illusions», JIB, 1998, vol. 9, n 3, p. 33-38.

${ }^{24}$ Voir la dramatique affaire France Télécom en France. Lors de la privatisation de l'entreprise et la nécessité de la rendre très rapidement plus performante, la direction déclina une politique de restructuration entre 2006 et 2010 destinée à obtenir 22000 départs et 10000 mobilités sur un effectif de 120000 salariés. La fixation de tels objectifs a nécessité de la part de la direction de déclencher un vaste et intraitable plan de restructuration donnant lieu à une vague de suicides sans précédent.
} 
L'enjeu est encore le décloisonnement de la santé au travail et de la santé publique. Le travail est une activité centrale des sociétés humaines, il peut ainsi impacter la santé en-dehors de la sphère du travail lorsque les conditions de travail sont à l'origine de maladies et d'accidents. Le travail «intervient indirectement par le type d'organisation de la société qu'il engendre» ${ }^{25}$. La protection de la santé des travailleurs est ainsi «l'affaire de la société entière dans le cadre d'un ordre public et social» ${ }^{26}$. Ce principe justifie et permet de comprendre la porosité de la frontière entre la santé publique et la santé des travailleurs, qui sont également des citoyens.

La reconnaissance de la santé mentale par le droit du travail (par la loi ou la jurisprudence selon le pays, par les textes internationaux) a permis de donner au concept de santé mentale une portée juridique, mais aussi symbolique car il prend en considération l'évolution de la conception de la santé au travail ${ }^{27}$. L'altération de la santé mentale imputable aux conditions de travail a, en effet, la nature d'un risque professionnel. La recrudescence des troubles mentaux liés aux conditions de travail actuelles ne se concilie pas avec la limitation des contours de l'accident du travail aux seules lésions corporelles. En d'autres termes, les altérations de la santé physique au travail (qui se voient) et les risques d'atteinte à la santé mentale au travail (qui ne se voient pas forcément) doivent être au même titre pris en compte. Si la santé se suffit par elle-même et transcende les frontières, le droit fondamental à la santé - comme le droit à la dignité dont l'une des composantes est le respect de la santé d'autrui - est inaliénable et ne peut s'arrêter à la frontière de la sphère du travail. Il n'est donc pas nécessaire de reconnaître un droit à la santé spécifique au travail.

Toutefois, en raison de la reconnaissance des liens entre santé au travail et santé publique, mais aussi de l'impact des organisations du travail et de leur mise en œuvre sur la santé des travailleurs, une approche holistique de la prévention des risques pour la santé au travail est devenue nécessaire. Aussi, pour favoriser cette approche et ce changement de paradigme, reconnaître formellement un droit fondamental à la santé au travail permettrait d'appuyer le principe que le droit fondamental à la santé s'applique aussi au travail et que nulle logique économique et de performance ne peut porter atteinte à la santé au travail. Ce processus ne saurait se heurter aux frontières du milieu professionnel.

Si la dignité à la santé doit être assurée en toute circonstance, notamment dans le cadre des relations de travail, le travailleur est d'abord une personne qui ne peut abdiquer sa santé en se mettant à la disposition d'un employeur. Toutefois, selon l'adage «à l'impossible nul n'est tenu», affirmer que les pouvoirs publics ont la charge d'assumer la protection de la santé semble utopique face à l'ampleur de la tâche que cela représente. La santé est un but à atteindre ${ }^{28}$. Assurer sa protection serait plus réaliste ${ }^{29}$, dans la sphère du travail également.

\footnotetext{
${ }^{25}$ Cassou B., «De la difficulté à construire une problématique santé dans le domaine du travail», Le Travail Humain, n 58, 4/1995, p. 355-358.

${ }^{26}$ Larger D., Miloch F., «Technique pénale et politique de prévention des accidents du travail: quelques thèmes de réflexion», Droit Social, juillet-août 1984, p. 498-504.

${ }^{27}$ Verkindt, Pierre-Yves (2005), Le droit du travail, Dalloz, Coll. Connaissance du droit, 2005, p. 132.

${ }^{28}$ Demichel A., Le droit de la santé, Les études Hospitalières, 1998, p. 13.

${ }^{29}$ Moreau J., «Le Droit à la santé», AJDA, 1998, numéro spécial, p. 185-190.
} 


\section{LE DROIT A LA PROTECTION DE LA SANTE AU TRAVAIL}

Parler de «droit à la protection de la santé» renvoie aux termes des textes de droits fondamentaux et des droits de l'Homme qui garantissent la protection des droits de tout à chacun. En outre, la notion de protection inclut une dimension préventive ayant pour objet et pour effet que la santé ne se dégrade pas (A). Cette évolution dans la conception de la santé au travail n'est pas sans poser des questions d'ordre éthique et de leur rapport au droit (B).

\section{Introduire de la prévention dans le droit à la santé au travail}

Le droit à la protection de la santé est un droit qui pourrait être qualifié de «droitsolidaire». Il concerne la communauté en général et «introduit le sentiment d’une solidarité qui unit les individus dans leur volonté de vivre ensemble» ${ }^{30}$. Le caractère solidaire du droit à la santé implique plusieurs débiteurs: d'abord l'Etat dans sa dimension d'Etat-providence, les professionnels de santé, l'individu lui-même en ce qu'il peut disposer des moyens d'assurer sa santé ${ }^{31}$ et, enfin, les entreprises. Il ne s'agit pas d'une obligation de résultat, mais bien d'une obligation de moyens consistant à mettre en œuvre un contexte social favorable à l'épanouissement de la santé de chacun et à mettre à disposition des moyens humains et matériels de façon à assurer la protection de la santé.

Les entreprises représentées par leur organe de direction sont donc débitrices du droit à la protection de la santé, ce qui permet de faire le lien entre la santé et le travail. Le droit de l'hygiène et de la sécurité est la traduction d'un droit à la protection de la santé au travail dont la protection de la santé physique des travailleurs a constitué «la pierre angulaire du droit du travail» ${ }^{32}$. Le terme de «santé» a cependant vocation à englober les aspects physiques et mentaux de la personne ${ }^{33}$. Or, le droit à la protection de la santé est qualifié de droit fondamental, à ce titre il doit s'appliquer au travail. Ainsi, en vertu du caractère fondamental du droit à la santé, le droit à la protection de la santé au travail est affirmé.

De la relation de travail entre l'employeur et le travailleur naissent deux risques différents. L'employeur met en jeu son patrimoine ou celui de l'entreprise tandis que le travailleur risque «sa peau» ${ }^{34}$. Le droit du travail s'est ainsi constitué dans l'optique de protéger cette dernière, d'abord en étendant le champ du principe de droit civil de la sécurité des personnes à l'entreprise, puis, ensuite, en prenant son indépendance au sein d'une réglementation spécifique de l'hygiène et de la sécurité ${ }^{35}$. L'aspect physique de la santé reste cependant dans la pratique du droit du travail prédominant. Affirmer expressément son aspect mental est fondamental de manière à lier sans contestation possible les acteurs compétents en matière de prévention des atteintes à la santé au travail à l'obligation de prévenir les risques pour la santé mentale au travail, au premier rang desquels figure l'employeur. La conséquence est aussi d'élargir le spectre aux conditions

\footnotetext{
30 Ibid.

31 Ibid.

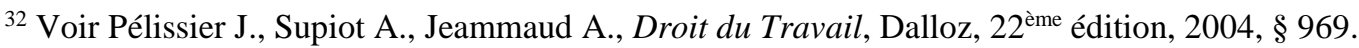

33 Bouty C., «Harcèlement moral et droit commun de la responsabilité civile», Droit Social, juillet-août 2002, p. 695-701.

${ }^{34}$ Supiot A., Critique du droit du travail, PUF, Coll. Les voies du droit, 1994, p. 68.

${ }^{35}$ Ibid.
} 
de travail, la protection de la «santé physique et mentale» concernant tous les pans du droit du travail car elle concerne les aspects organisationnels.

Le droit à la protection de la santé - la santé renvoyant au plus intime de la personne - est susceptible de justifier des restrictions apportées à d'autres principes de même valeur constitutionnelle. Ainsi, au nom des impératifs de santé publique, le droit de propriété, le droit de grève ou encore la liberté d'entreprendre s'appliquent dans la limite où ils ne portent pas atteinte à la santé de la personne ${ }^{36}$. Le droit à la protection de la santé au travail, entendue dans un sens global, émerge de l'affirmation d'une obligation générale de prévention et de sécurité à la charge de l'employeur et confirmée par la directive-cadre du 12 juin 1989 et qui va au-delà du respect des normes techniques et de la mise en œuvre d'organe de protection de la santé au travail. L’employeur doit intégrer dans sa politique de gestion les aspects liés à la santé et à la sécurité. Du côté des travailleurs, ces derniers doivent prendre soin de leur sécurité et de leur santé ainsi que de celles des autres personnes du fait de leurs actes ou de leurs omissions au travail.

Les inquiétudes nées de l'emploi important de la main-d'œuvre enfantine et féminine dans des conditions physiques et morales des plus éprouvantes vont justifier l'édiction d'une législation spécifique propre à réglementer les conditions de travail. $\mathrm{Au}$ fil de l'histoire du droit du travail, le droit à la protection de la santé au travail va se diviser en trois segments. D'une part est mise en place une réglementation qui impose des obligations en matière d'hygiène, de santé et de sécurité aux employeurs et aux salariés ; d'autre part sont institués des organismes permettant une adaptation aux multiples problèmes internes aux entreprises. Enfin, des droits sont reconnus aux travailleurs de manière à leur attribuer des moyens d'action autonomes.

Ces trois segments contiennent les éléments caractérisant l'émergence et l'affirmation du droit à la protection de la santé au travail dans la deuxième moitié du $X^{X} X^{\text {ème }}$ siècle, comme en France à travers la protection des enfants et des femmes au travail, l’hygiène et la sécurité dans les établissements industriels. Ces grands textes sociaux posent les premiers jalons de la protection de la santé et de la sécurité des travailleurs jusqu’à la reconnaissance de l'obligation générale de sécurité pesant sur l'employeur et le chef d'entreprise. Dit d'une autre manière, l'importance accordée à la reconnaissance d'une obligation générale de sécurité de l'employeur par la jurisprudence est l'expression d'un droit à protection de la santé au travail reconnu aux salariés.

L’obligation générale de sécurité, mais aussi l’obligation générale de prévention, ne sont pas les seules facettes de la reconnaissance du droit à la protection de la santé au travail. En effet, la législation du travail y ajoute des impératifs particuliers tenant à l'obligation de formation et à des droits autonomes reconnus au travailleur en situation de danger grave et imminent que sont le droit de retrait et le droit d'alerte.

La reconnaissance du droit à la protection de la santé au travail est la reconnaissance d'une conception extra-patrimoniale des relations de travail permettant le respect de l'intégrité du travailleur au travail. La personne du travailleur est placée au centre de la relation de travail et devient l'objet partiel du contrat de travail avec la prestation de travail. L'esprit du texte de la directive-cadre du 12 juin 1989 est notamment d'adapter le travail à l'homme qui devient un concept novateur en s'opposant à la maxime de l'organisation scientifique du travail qui prône «l'adaptation de l'homme au travail». Cette inversion dans la conception du travail et de son organisation se réalise grâce à la

${ }^{36}$ Ibid. p. 69; Villiers (de) M., Renoux T.-S., Code Constitutionnel commenté et annoté, Litec, 2001, p. 240 ; Moreau J., «Le Droit à la santé», AJDA, 1998, numéro spécial, p. 185-190. 
prise en compte de l'amélioration des conditions de travail. Au lieu d'adapter l'homme physiquement aux contraintes de production, le travail est envisagé de façon à rendre les conditions physiquement et mentalement acceptables. Cette approche participe à l'humanisation du travail, à retrouver l'humain qui disparait derrière des normes d'organisation du travail. Aborder la question sous l'angle éthique prend alors du sens aujourd'hui.

\section{Droit à la santé au travail et approche éthique}

Le travail est une activité centrale au sein des sociétés humaines. Il peut ainsi influencer la santé directement lorsque les conditions de travail sont à l'origine de maladies et d'accidents. De même, les normes juridiques apportent des garanties dont le but est de protéger la santé au travail et en dehors du travail. Toutefois, l'évolution du travail et ses conséquences amènent à interroger la responsabilité de chacun au sein d'un collectif de travail, la santé et le bien-être qui en découlent sont l'affaire de tous les acteurs de l'entreprise et pas seulement des préventeurs et des dirigeants. L'objectif est d'encourager le développement d'une organisation du travail promotrice de santé, sur la base de valeurs, entre autres, de coopération et de justice.

Cette approche remet au centre le débat des liens ou différences entre morale, éthique et droit. Le but est d'appréhender les façons dont éthique et santé au travail s'articulent et même se complètent de manière à ouvrir une nouvelle voie pour appréhender juridiquement la santé au travail au regard de l'évolution économique, politique et sociale du monde d'aujourd'hui. Le cadre de référence ne sera pas celui de la morale, mais celui du droit en lien avec la santé au travail. Ainsi, le «juste» que nous cherchons à atteindre est celui qui est conforme au respect de la règle. L'éthique conçue ici pour fonder nos recherches n'est pas un ensemble de valeurs ni de principes, mais une réflexion argumentée en vue d'agir efficacement au service du bien-être au travail. Cette réflexion permet aussi d'interroger le sens et l'esprit des décisions et des organisations du travail au regard du respect des règles.

L'accent porte sur le soutien à la prise de décision face à des enjeux concrets relatifs aux processus décisionnels, sur les valeurs et les principes mis en jeu et leurs rapports entre eux au regard du cadre de référence qui constitue le droit. L'esprit qui accompagne l'édiction des normes juridiques et le sens de leur application ne sont pas hermétiques à la juxtaposition de valeurs éthiques. Par exemple, parler d'humain dans le contexte de travail s'oppose à la réification de la personne du travailleur niant son humanité dans la manière de gérer le travail. L’approche éthique va guider l'application des normes juridiques relatives aux pouvoirs de l'employeur en matière de direction et de santé au travail. L'éthique dans le milieu professionnel renvoie au sens des décisions prises, aux valeurs et à l'esprit qui leur sont associés. Elle justifie l'action des acteurs du monde du travail aux niveaux individuel et collectif, mais aussi leur responsabilité. L'objectif est ici d'amener les futurs acteurs du travail à mieux appréhender le monde du travail dans lequel ils vont évoluer.

Le droit montre le chemin, la voie à suivre et fixe les limites tout en garantissant la liberté de chacun. À travers une approche éthique, il s’agit de réinterroger les normes juridiques du travail et de la santé au travail, de mieux comprendre les droits et obligations qui en découlent. L’enjeu est aussi de répondre à la recherche d’adhésion et d'acceptation 
sociale du droit ${ }^{37}$ de la santé et de la sécurité au travail comme de questionner le contrôle et la sanction indissociables des politiques de santé et de sécurité au travail, mais encouragés ${ }^{38}$ ou remis en question ${ }^{39}$.

Travail et santé font système, ainsi une approche holistique est aujourd'hui de mise. Toutefois, avant de la décréter, des questions préalables sont à clarifier à la lumière d'une approche éthique destinée à renouveler la manière d'envisager la santé au travail:

- Au regard de l'évolution de l'approche de la santé au travail qui est de plus en plus associée à la «performance globale» ${ }^{40}$, quelle est la définition de la «performance» sur laquelle s'appuyer pour définir des politiques de protection de la santé au travail?

- Quelles valeurs place-t-on dans le travail et quel mode de vie en retour en retire-t-on?

- Peut-on interroger les logiques économiques au regard de la santé au travail? Quelle est la place de l'humain dans ce contexte?

Enfin, les normes juridiques ont-elles la capacité et la force pour faire du travail un facteur de bien-être? L'approche par l'angle éthique est une des pistes à explorer pour répondre positivement à cette question. À travers la réflexion éthique, le but est de créer les conditions d'un engagement responsable, de modes d'organisation du travail humain, de répondre au principe d'adaptation du travail à l'homme, de favoriser le bien-être au travail.

\footnotetext{
${ }^{37}$ Voir Atias C., Philosophie du droit, PUF, coll. Thémis, 1999, p. 168.

38 Voir Rapport «Frimat», Mission relative à la prévention et à la prise en compte de l'exposition des travailleurs aux agents chimiques dangereux, remis à la ministre du travail Murielle Pénicaud le 29 août 2018, 47 p.

${ }^{39}$ Voir Lecocq C., Dupuis B., Forest H. avec l'appui d'Hervé Lanouzière, Santé au travail: vers un système simplifié pour une prévention renforcée, rapport fait à la demande du Premier Ministre et remis le 28 août 2018, 174 p.

${ }^{40}$ Voir les rapports dits «Lecocq» sur la réforme du système de santé au travail salarié et dans la fonction publique, Lecocq C., Dupuis B., Forest H. avec l'appui d’Hervé Lanouzière, Santé au travail: vers un système simplifié pour une prévention renforcée, rapport fait à la demande du Premier Ministre et remis le 28 août 2018, 174 p.; Lecocq C., Coton P., Verdier J.-F., Santé, sécurité, qualité de vie au travail dans la fonction publique: un devoir, une urgence, une chance, rapport fait à la demande du Premier Ministre et remis le 28 octobre 2019, 113 p.
} 